\title{
RESEARCH
}

\section{Analysis of the global situation of COVID-19 research based on bibliometrics}

\author{
Fan Yang ${ }^{1,2+}$ (1), Shuaijie Zhang ${ }^{1,2+}$, Qing Wang ${ }^{1,2}$, Qi Zhang ${ }^{2}$, Junming Han ${ }^{1,2}$, Lijie Wang ${ }^{1,2}$, Xinying Wu $u^{1,2}$ \\ and Fuzhong Xue 1,2* $^{*}$
}

\begin{abstract}
With the rapid global spread of the COVID-19 pandemic, researchers have contributed several important advances. The WHO and countries with severe outbreaks have developed diagnosis and treatment guidelines. Here, we analyze the current transformation and application of scientific research to global epidemic prevention and control. We described and analyzed current COVID-19 research from the perspectives of international cooperation, interdisciplinary cooperation, and research hotspots using a bibliometric clustering algorithm. Using the diagnosis and treatment guidelines of the WHO and the United States and China as examples, we evaluate the transformation of scientific results from basic research to applications. Scientific research results that have not yet been incorporated into these guidelines are summarized to encourage updates and improvements by applying scientific research to prevention and control. COVID-19 has fostered interdisciplinary cooperative research, and the current results are mainly focused on the origin, epidemiological characteristics, clinical research, and diagnosis and treatment methods for the virus. Due to the ongoing publication of new research, diagnosis and treatment guidelines are constantly improving. However, some research gaps still exist, and some results have not yet been incorporated into the guidelines. The current research is still in the preliminary exploratory stage, and some problems, such as weak international cooperation, unbalanced interdisciplinary cooperation, and the lack of coordination between research and applications, exist. Therefore, countries around the world must improve the International Public Health Emergency Management System and prepare for major public health emergencies in the future.
\end{abstract}

Keywords: COVID-19, Bibliometrics, Diagnosis and treatment guidelines, Global epidemic

\section{Introduction}

In early 2020, the COVID-19 outbreak began in China and quickly spread around the world. This epidemic has tested the construction of global emergency management and major epidemic prevention control mechanisms. Facing the grim situation of the spread of the epidemic throughout the world, researchers have conducted multicountry, multidisciplinary and comprehensive joint research studies to examine the problems related to the epidemic that require solutions.

The epidemic prevention and control model, which has been transformed by research and use, has played

\footnotetext{
*Correspondence: xuefzh@sdu.edu.cn

†Shuaijie Zhang contributed equally to this work with Fan Yang.

${ }^{2}$ Institute for Medical Dataology, Shandong University, Jinan 250002,

Shandong, China

Full list of author information is available at the end of the article
}

an important role in this epidemic. The WHO notes that research can be applied by "taking what we have learned so far about the virus and translating that knowledge into strategic action that can guide the efforts of all national and international partners to support national governments". Therefore, under the guidance of the WHO, most countries have adopted the strategy of scientific research and the epidemic prevention model. Countries with severe outbreaks have developed treatment plans based on the existing results of scientific research and national conditions. Researchers are obtaining a new understanding of COVID-19, and the diagnosis and treatment guidelines of various countries are constantly changing and improving based on new results from scientific research. In China, the country with the first outbreak, the Chinese government has issued seven versions of the diagnosis and treatment guidelines for the treatment of 
patients based on research findings obtained over time. This agile strategy also illustrates the important role of scientific research in epidemic prevention.

Although the combination of scientific research and clinical treatment has played a role in preventing and controlling this outbreak, some problems associated with the prevention and control of the current epidemic still exist. First, scientific research achievements are not summarized systematically. Although researchers have made positive progress in COVID-19 research, these scientific achievements are not summarized and classified systematically, which is not conducive to understanding the research status and research trends, and thus, the results are unable to be promptly applied in practice. Second, further in-depth and expanded scientific studies of epidemic prevention and control are needed. For example, the host, transmission route, pathogenic mechanism, and drug development have not yet been clarified. Additionally, gaps and unexplored areas still exist in some aspects of scientific research and development that determine the progress of epidemic prevention and control. Only the prompt elimination of these gaps and unexplored area will allow research to become more conducive to preventing and controlling the pandemic. Third, scientific research and clinical treatments should be coordinated, and epidemic prevention and control plans should be assessed and adjusted in a timely manner. Government departments should remain updated on the scientific research status of COVID-19, apply the research results, and update their response strategies according to trends in the development of the epidemic. In summary, scientific research and practical experience complement each other and should be combined. Scientific research findings support prevention and control work, and prevention and control work provides practical experience to inform scientific research.

Therefore, we used bibliometrics and clustering algorithms to analyze the COVID-19 literature and representative diagnosis and treatment guidelines, better respond to major global public health emergencies in the future, improve the major epidemic prevention and control regulations, and develop emergency treatment management methods. We systematically summarized the research status of COVID-19 to examine the research trends and hotspots. Then, we analyzed the diagnosis and treatment guidelines of the WHO, the US and China and identified research gaps based on the diagnosis and treatment guidelines used in practice that suggest new directions for scientific research. In addition, we summarized the scientific research results that have not yet been incorporated into the diagnosis and treatment guidelines and organized them to promote the establishment of a cooperative mechanism of scientific research and clinical treatment.

\section{Research in context}

Evidence available before this study

COVID-19 is spreading all worldwide, but few reports have described the current status of related scientific research. We searched PubMed on April 9th using the search terms ("2019-nCoV" OR "SARS-CoV-2" OR "COVID-19" OR "novel coronavirus" OR "coronavirus") AND ("scientific research achievements" OR "research achievements" OR "guidelines" OR "guidance" OR "research situation" OR "science") for studies published within 1 year. We also evaluated an editorial, "Science in the time of coronavirus", which noted that after the outbreak, the number of papers analyzing COVID-19 increased significantly. However, no studies have summarized COVID-19 research and transformation.

\section{Added value of this study}

We describe and analyze various versions of diagnosis and treatment guidelines in relation to the present status of COVID-19 research to effectively promote scientific research that will help prevent and control the epidemic, to remedy the lack of analysis of the current situation of scientific research, and to inform the management of key problems related to the prevention and control of the epidemic. This study will help improve the standards for the prevention and control of major epidemics and the methods for managing emergency treatment.

\section{Implications of all the available evidence}

We summarize the current situation of COVID-19 scientific research and provide a reference for updating diagnosis and treatment guidelines. In addition, we note gaps in the diagnosis and treatment guidelines that warrant new scientific research.

\section{Methods}

We have collected 1892 papers analyzing COVID-19 from the Chan Zuckerberg Initiative, ${ }^{1}$ medrxiv, ${ }^{2}$ biorxiv, ${ }^{3}$ Web of Science, ${ }^{4}$ and PubMed. ${ }^{5}$ The WHO diagnosis and treatment guidelines, American diagnosis and treatment guidelines, and Chinese diagnosis and treatment guidelines were all obtained from the official websites of these health organizations.

\footnotetext{
${ }^{1}$ https://chanzuckerberg.com/covid-19/.

2 https://connect.medrxiv.org/relate/content/181.

3 https://connect.biorxiv.org/relate/content/181.

${ }^{4}$ https://mjl.clarivate.com/search-results.

${ }^{5}$ https://pubmed.ncbi.nlm.nih.gov/.
} 
We collected abstracts, keywords, and author information (including the country and main field of study) from the 1892 papers using crawlers. Text preprocessing was performed on the collected data. We started by removing non-text parts of the data with the regular expression re module of Python version 3.6 (Python Software Foundation, Beaverton, OR, USA), segmented the words, deleted the stop words, and restored the word forms. All English words were converted to lowercase to reduce the number of words. Finally, we used the word2vect CBOW model for feature processing, which is shown in Algorithm 1. The CBOW model is composed of an input layer, a projection layer, and an output layer. In the input layer, word vectors of $\mathrm{C}$ words before and after the word $\mathrm{w}$ were constructed for a total of $2 \mathrm{C}$ word vectors. For the projection layer, the $2 \mathrm{C}$ vectors of the input layer are summed. The output layer constructs a binary tree, and the words that appear in the corpus are used as leaf nodes. The weight of each node represents the frequency of the feature words in the text set.

Vosviewer was used to analyze the research hotspots and obtain the preliminary visualization results, and then drawing software was used to simplify the preliminary visualization results. The principle of using Vosviewer to construct the initial clustering graph is described below. (1) First, the similarity was measured. The correlation strength was used to calculate the similarity between two items $i$ and $j$. And $s_{i j}$ stands for the similarity that is defined as $s_{i j}=\frac{c_{i j}}{w_{i} \times w_{j}}$, where $c_{i j}$ denotes the total number of co-occurrences of items $i$ and $j$, and $w_{i}$ and $w_{j}$ denote the total number of occurrences of items $i$ and $j$, respectively. (2) Then, the Label-based method clustering algorithm was used to generate a clustered label map. The principle of the algorithm is to minimize the weighted sum of the squared Euclidean distances between all pairs of items. A higher level of similarity between two items increases the weight of their squared distance in the summation. The algorithm of Label-based method is $V\left(\boldsymbol{x}_{1}, \ldots, \boldsymbol{x}_{n}\right)=\sum_{i<j} s_{i j}\left\|\boldsymbol{x}_{i}-\boldsymbol{x}_{j}\right\|^{2}$, where the vector $\boldsymbol{x}_{i}=\left(x_{i 1}, x_{i 2}\right)$ denotes the location of item $i$ in a twodimensional map and $\|\cdot\|$ denotes the Euclidean norm.

The co-occurrence network map was used to establish a national cooperation network. First, an adjacency matrix was built to map the relationship between nodes. Nodes represent countries, and when authors co-author the same document, then a connection is established between the authors' country. The names of all countries were entered in rows and columns. When two countries appeared in the author information table of the same document, the number 1 was placed at the intersection between the two; otherwise, the number 0 was placed at the intersection. If the authors collaborated multiple times, the number was equal to the total number of coauthored papers. The size of the node was determined by the proportion of national co-authored papers to the total number of papers. Then, the connection weight was calculated, namely, the proportion of the actual number of connections between countries in the network to the total number of connections, and the width of the edge was set according to the connection weight. Finally, the country nodes were mapped to the map.

The proportion of research results in each country was calculated based on the proportion of published studies in the total literature. Regarding subject participation, all authors were initially classified according to their major field of study. When a document involves multiple 


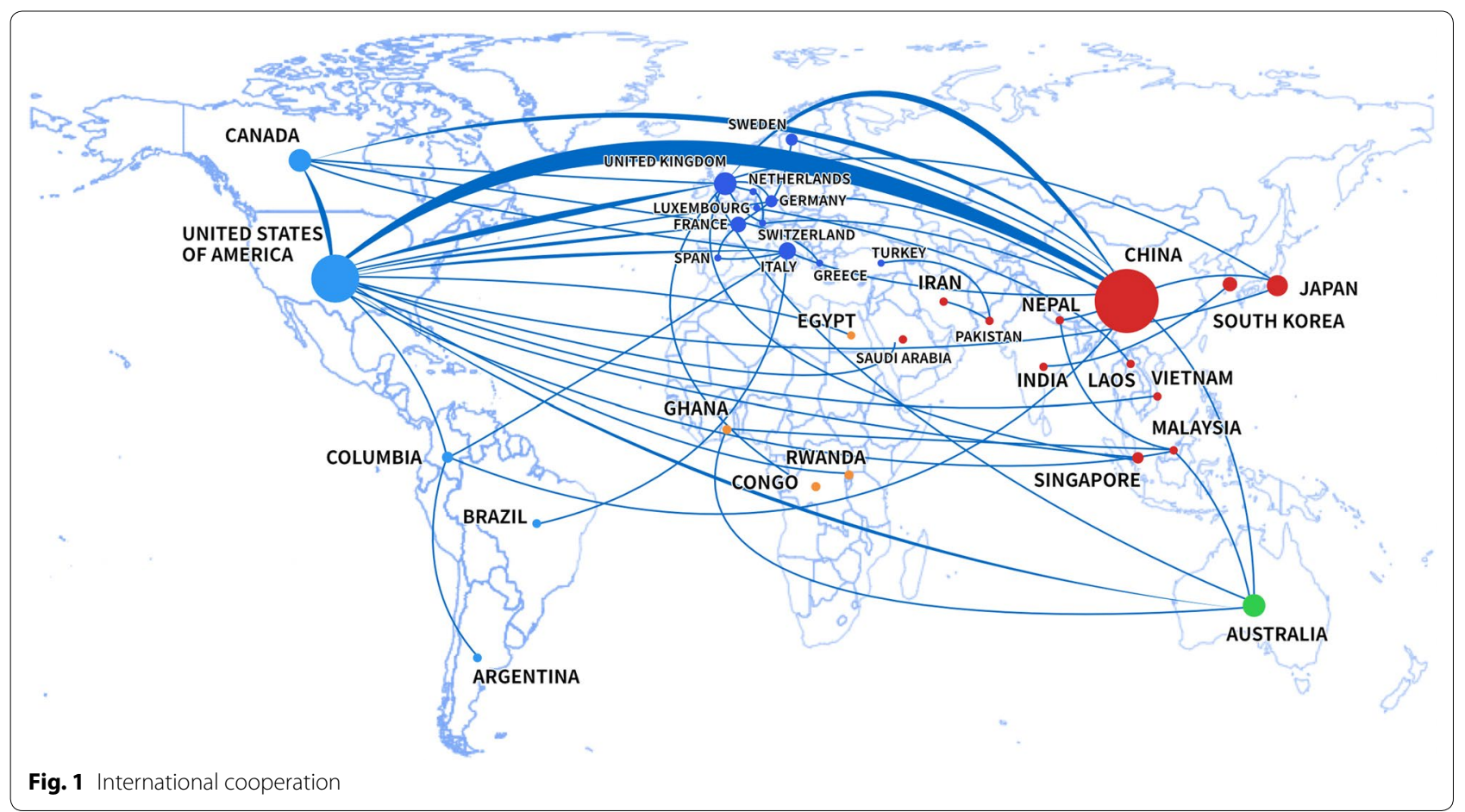

disciplines, we added the number of disciplines involved for each author. Finally, the participation ratio of each disci-

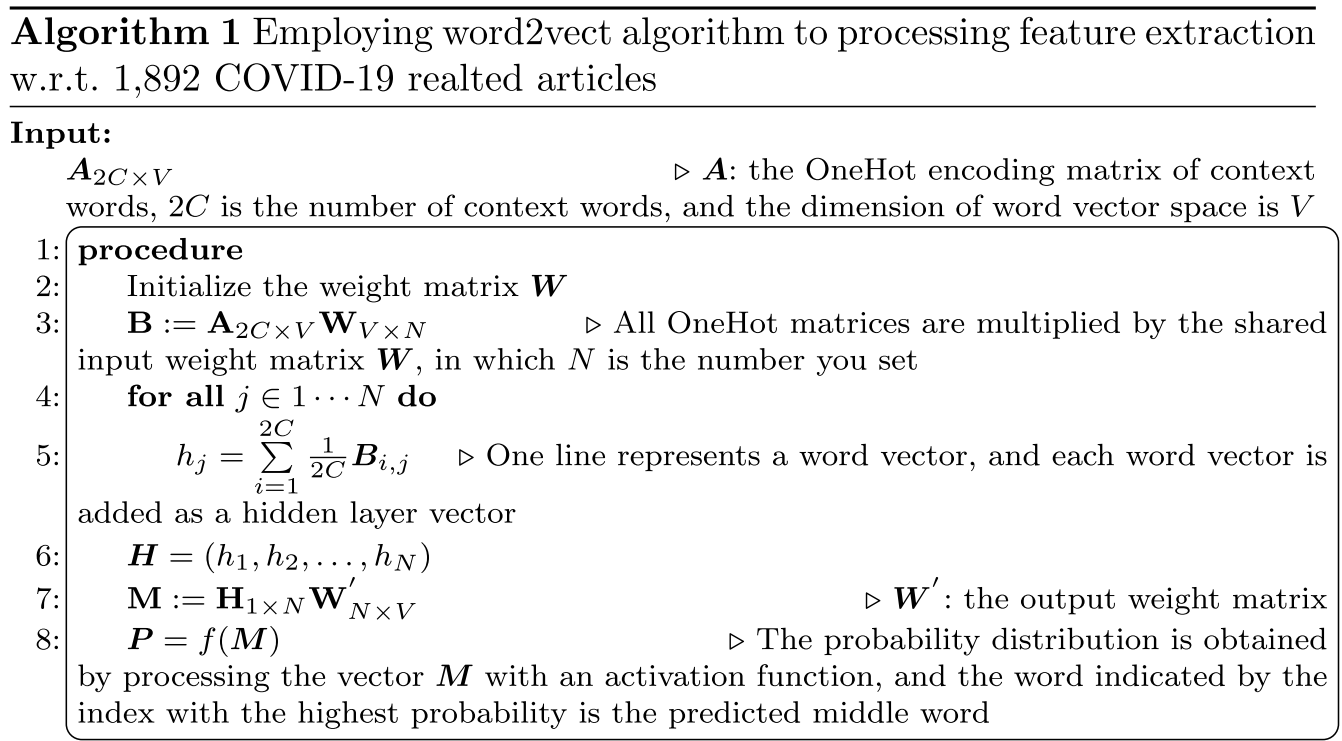

Output: word vector $\boldsymbol{P}$

pline in all disciplines was calculated, and this ratio was defined as discipline participation. 


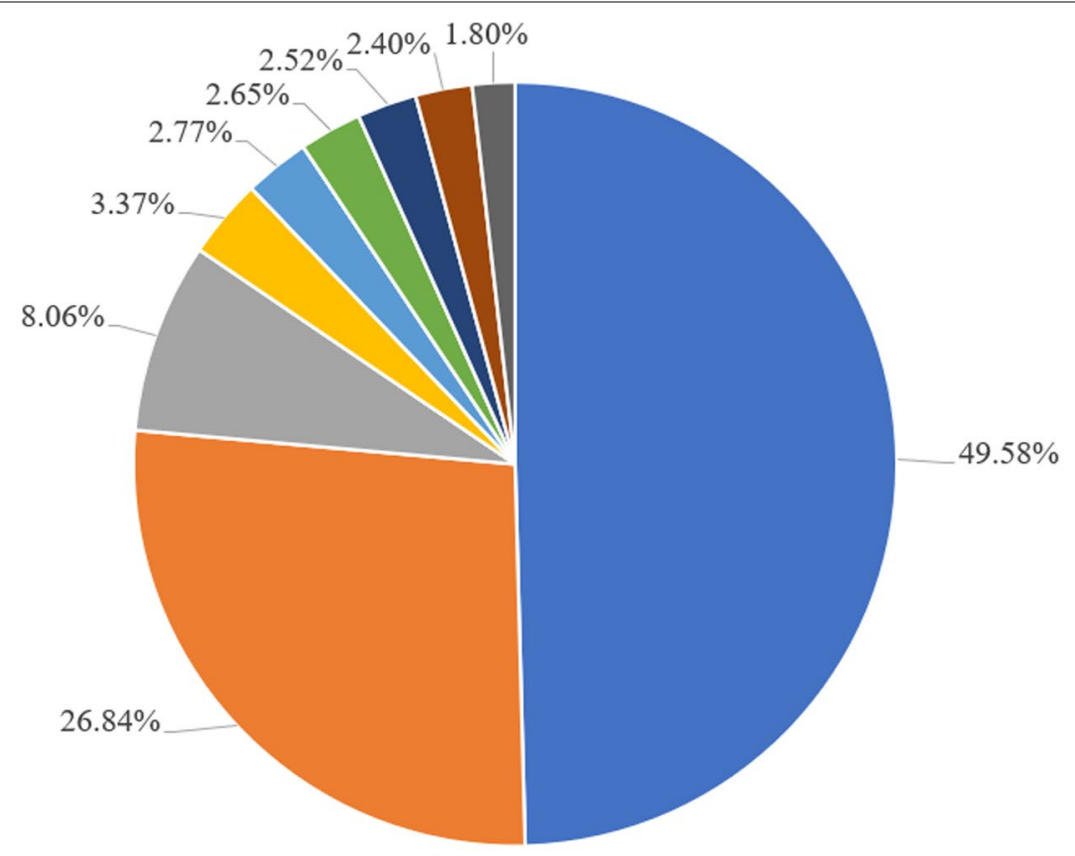

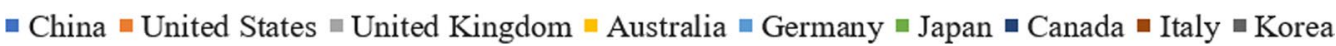

Fig. 2 Proportions of research results

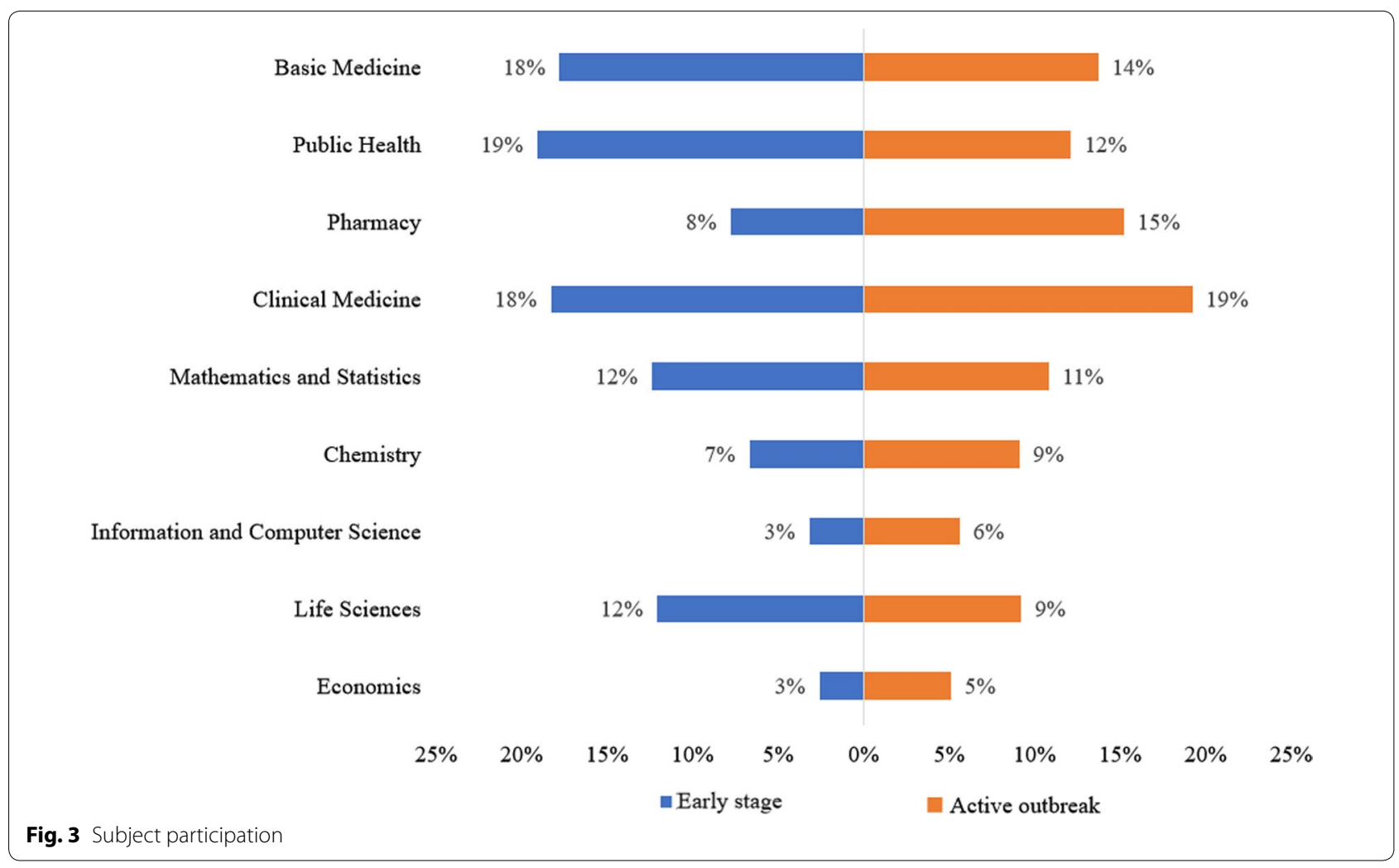




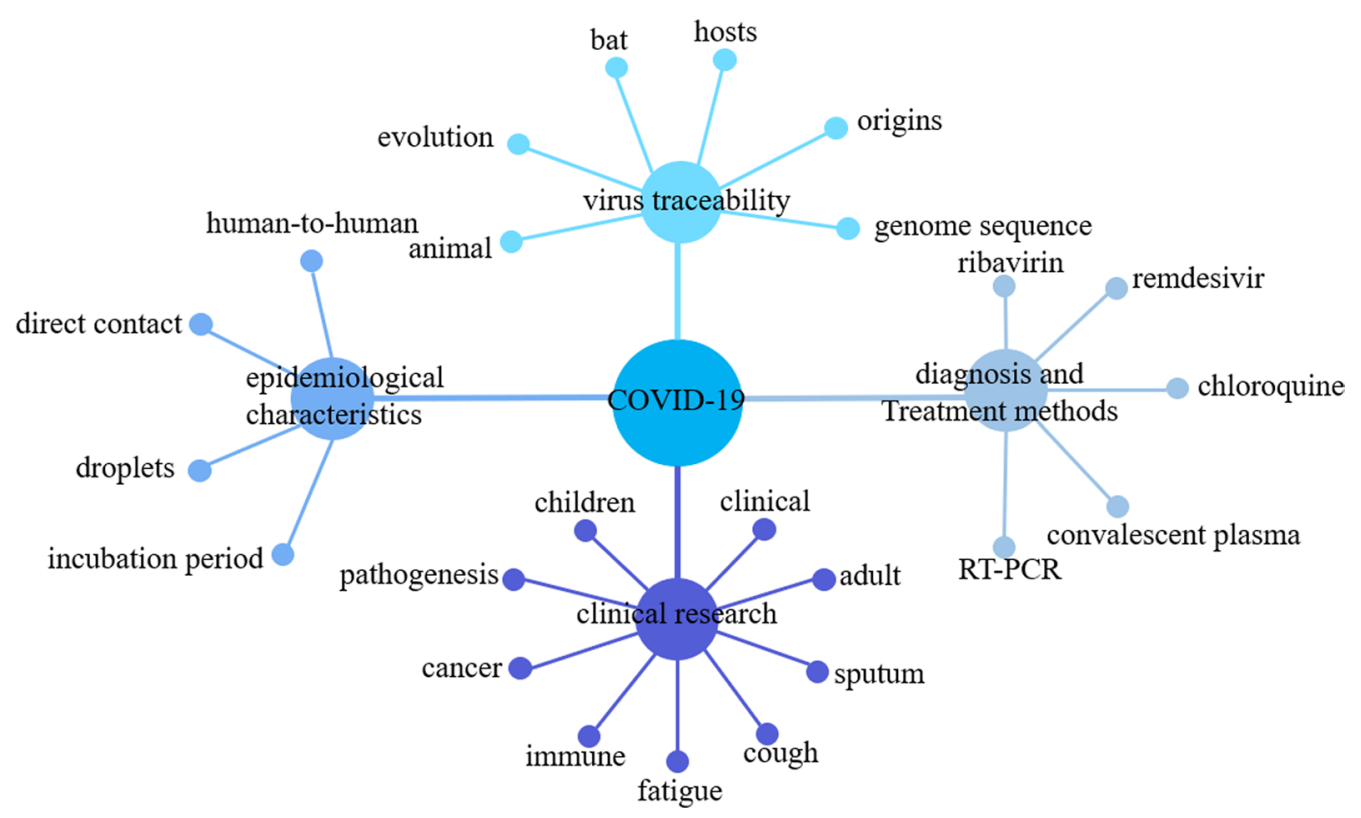

Fig. 4 Subject participation

\section{Results}

\section{Analysis of international research partnerships}

In the knowledge map of international research cooperation (Fig. 1), countries actively participating in COVID19 are identified. The lines between the nodes represent connections between countries, and the thickness of each line represents the cooperation intensity between those countries. As shown in the figure, the current research on COVID-19 mainly involves international cooperation between China and the US. China has formed cooperative relations with the US, Germany, the UK, Italy, Colombia, Canada, and Japan. In addition, the US has cooperated with Japan, Canada, the UK, Italy, Australia and France. Among these countries, China has the closest cooperation with the US. Currently, although many countries have cooperative relationships, their cooperation is weak, and research on COVID-19 is still dominated by the internal research conducted in each country. Moreover, cooperation in regions with less developed medical facilities, such as Africa and South America, is not ideal. These regions should focus on advancing preparations for prevention and control to avoid the occurrence and spread of the epidemic.

In addition, we used the number of published papers as an index of national research achievements. As shown in Fig. 2, most achievements in COVID-19 researchers have occurred in China, the US, and the UK. China accounts for $48.70 \%$ of the global research results, and the US accounts for $26.36 \%$. As the country where the initial outbreak occurred, China has published the most studies on
COVID-19. Some countries have had severe outbreaks, such as Italy, Spain, Germany, Iran, and France, but their scientific research process has not been coordinated with the development of the epidemic, and results are lacking from these countries. The combination of scientific research with clinical experience is crucial to curb the development of the epidemic as soon as possible.

\section{Analysis of interdisciplinary cooperative relationships}

We analyzed the cooperation among disciplines at the beginning of the outbreak and in later stages (Fig. 3). Scientists in the fields of clinical medicine, basic medicine, public health, pharmacy, chemistry, life sciences, information and computer science, economics, and mathematics and statistics have all contributed to the study of COVID-19. At the beginning of the epidemic, all these disciplines quickly worked together to conquer the epidemic. Among these disciplines, the participation rates of scientists in clinical medicine, basic medicine, and public health were relatively high in the early stage of the epidemic, and the participation rates of scientists in pharmacy and chemistry increased at the peak of the epidemic compared with the early stage of the epidemic.

In the early stages of the epidemic, because researchers lacked awareness of COVID-19, they first learned about COVID-19 by studying the epidemiological characteristics of the virus, such as traceability and transmission routes, and examining the susceptible populations. Then, researchers observed the clinical characteristics of patients, such as lung computed tomography imaging 


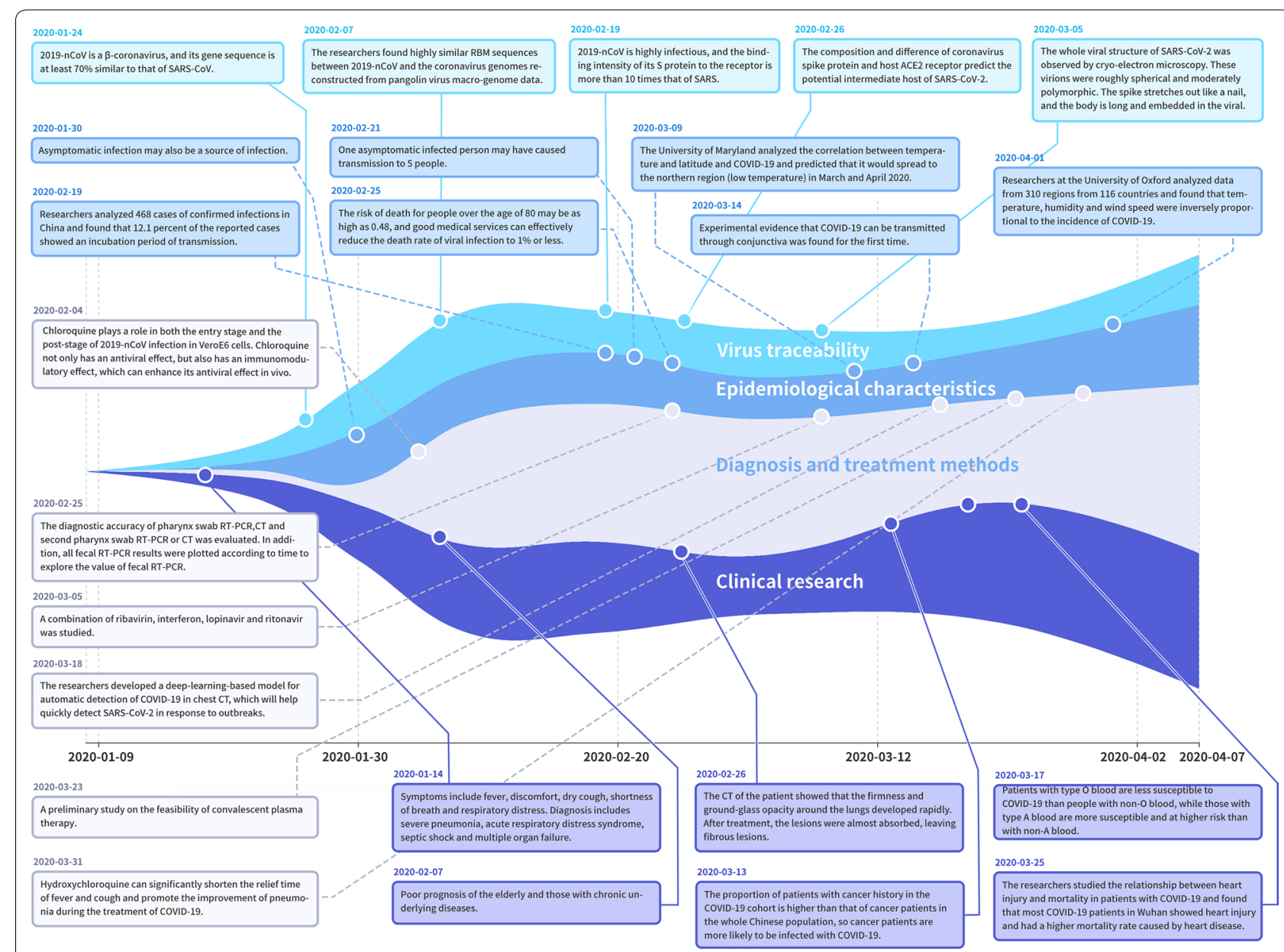

Fig. 5 Major research results

characteristics and changes in functional metabolism, to further grasp the pathogenesis and provide a reference for the identification of effective treatments. Thus, in the early stages of the epidemic, scientists in the fields of public health, clinical medicine, and basic medicine had relatively high levels of participation compared to the other disciplines. In addition, as shown in the figure, the participation of scientists involved in the fields of chemistry and pharmacy increased rapidly in the peak epidemic period. These results show the spread of the epidemic and the early, preliminary understanding of COVID-19, followed by the combination of efforts from biochemists and pharmacists to identify effective therapeutic drugs. The participation of mathematicians and statisticians was relatively stable, and scientists in these disciplines were primarily integrated with public health scientists in predicting outbreaks and incubation periods. Information and computer scientists mainly provide support for the prevention and control of the epidemic through artificial intelligence and other technologies in intelligent screening, diagnosis, triage, epidemic prediction and analysis, screening and early warning of people with a high temperature in large places, among other aspects. The spread of the disease has also affected the global economy, and thus economic research has increased with the outbreak.

\section{Research hotspots and dynamic analysis}

Key co-occurrence maps with four clusters were drawn to represent the main research directions (Figs. 4, 5): virus traceability, epidemiological characteristics, clinical research, and diagnosis and treatment methods.

The keywords involved in virus traceability research are hosts, animal, evolution, bat, origins, and genome sequence. The new coronavirus is a $\beta$-coronavirus whose genetic sequence is at least $70 \%$ similar to SARS$\mathrm{CoV}$. Zhu et al. (unpublished) and Song et al. [8] used electron microscopy and cryo-electron microscopy to directly observe 2019-nCoV. Zhu et al. (unpublished) and Wong et al. (unpublished) studied the similarities in the 
genomic sequences among viruses, searched for virus hosts and found that bats may be the source host and pangolins may be the intermediate host. Other researchers studying potential hosts have reported different conclusions. For example, Lam et al. (unpublished) found that the coronavirus in pangolin samples was $97 \%$ similar to 2019-nCoV. Ji et al. [5] indicated that snakes may be the host; Guo et al. (unpublished) used a deep learning algorithm to sequence the genomes of a variety of vertebrates, compared them with $2019-\mathrm{nCoV}$, and found that mink might be an intermediate host.

Research into the epidemiological characteristics involves the following keywords: human-to-human, direct contact, droplets, and incubation period. The infection sources of COVID-19 are mainly patients with COVID-19 and people with asymptomatic infections. Thus, the incubation period and asymptomatic infection spread have become research hotspots. Researchers analyzed 468 confirmed infections in China and found that $12.1 \%$ of the reported patients showed transmission during the incubation period [3]; Bai reported that one asymptomatic infected person may have caused transmission to five people [1]. Currently, evidence for both latency and asymptomatic transmission is limited. In addition, other studies have shown that the transmission routes of COVID-19 are mainly droplet transmission and close contact, and the population is generally susceptible to COVID-19.

The clinical research field involves the following keywords: clinical, sputum, cough, fatigue, immune, adult and children, pathogenesis, cancer, etc. The common symptoms of COVID-19 are fever, discomfort, dry cough, and shortness of breath [4, Zeng et al. (unpublished)] , and the $\mathrm{CT}$ features of the patients lungs show the rapid development of peripulmonary lesions and groundglass opacities [11]. According to Wang and Zhang [9], patients with cancer are more susceptible to COVID19. As shown in the study by Zhao et al. (unpublished), individuals with type $\mathrm{O}$ blood are less susceptible to COVID-19, while individuals with type A blood are more susceptible and at higher risk.

Keywords in the field of diagnosis and treatment tools include RT-PCR, ribavirin, remdesivir, chloroquine, convalescent plasma, etc. In addition to developing diagnostic reagents, researchers have developed a deep learning-based chest CT model for the automatic detection of COVID-19 (Zheng et al. unpublished). In terms of the clinical treatment, researchers have not yet identified a specific treatment for COVID-19, but the disease has been treated with antiviral drugs, such as $\alpha$-interferon [7], lopinavir [6], ritonavir [6], ribavirin [2], chloroquine phosphate [10], and remdesivir [6].

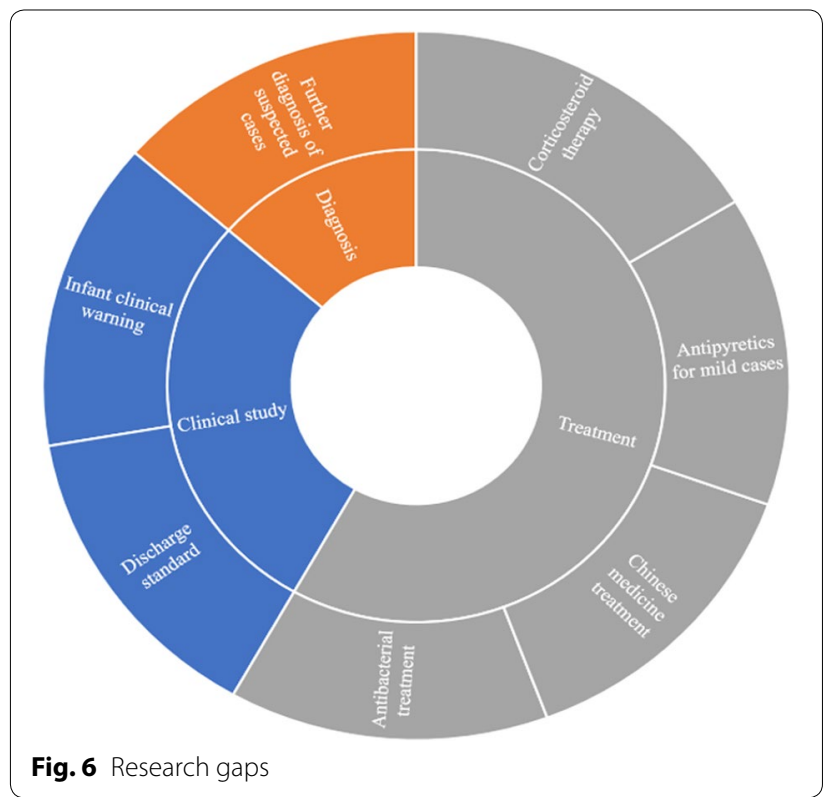

\section{Diagnosis and treatment program and an analysis of the supporting hypothesis}

Considering the global research results on COVID-19, we present the basis of the diagnosis and treatment guidelines in the Supplementary Material (Table 1), and these guidelines are divided into four aspects: virus traceability, epidemiological characteristics, clinical research, and diagnostic and treatment tools. As shown in the table, most of the guidelines are supported by relevant scientific hypotheses, reflecting the scientific research mode in which basic research is transformed into applications.

\section{Comparative analysis of the WHO, American, and Chinese medical guidelines}

As the first country to experience the COVID-19 outbreak, China has released seven versions of the diagnosis and treatment guidelines to date based on clinical experience and global scientific research results. The second edition of the WHO clinical management guidelines refers to the seventh edition of the Chinese guidelines for classification management and clinical treatment, while the American medical guidelines refer to the WHO. In terms of specific contents, the WHO provides detailed suggestions for protection during sample collection, the prevention of complications, management of special populations and management of clinical research. Meanwhile, China provides specific references in terms of treatment drugs, severe and critical warning indicators, and the management and treatment of critically ill patients. In terms of other aspects, China should actively refer to the WHO guidelines for updates. 
In addition, in terms of laboratory diagnosis and based on the situation of "multiple negatives and one positive", the WHO recommends the collection of samples from the LRT for retesting as much as possible, while the Chinese guidelines emphasize detection in multiple samples from multiple sites. The effectiveness of these two different methods requires further study and exploration. The American medical guidelines also provide specific operating procedures for sample collection, storage, transportation, and laboratory diagnosis based on the WHO guidelines. The WHO, the US, and China diagnosis and treatment guidelines frequently refer to each other, although the specific contents suitable for the epidemic in each country are also present. Countries should communicate with the WHO in a timely manner and update their diagnosis and treatment guidelines to help promote the global epidemic prevention and control process. Details are provided in the Supplementary Material (Table 2).

\section{Research gaps in medical guidelines}

Some components of the diagnosis and treatment guidelines are not based on science. We present these gaps and unexplored areas in the existing studies with the aim of spurring discussion and study by researchers, which are presented in Fig. 6.

\section{Further improvement of the medical guidelines}

Through the analysis of COVID-19-related papers, some scientific results were not reflected in the medical guidelines, and we present these results in a table. These findings, which have not yet been incorporated into the treatment program, may provide a reference for countries with severe epidemic situations and help to further improve the treatment program in each country or region. Details are provided in the Supplementary Material (Table 3).

\section{Discussion}

During this outbreak, some problems related to the prevention and control of major global epidemics have been exposed. Countries around the world should learn from the advanced international mechanisms for the prevention and control of major epidemics and the public health emergency management system in a timely manner. A summary of the existing research results and practical experience will provide useful insights for future public health emergencies.
Improve international cooperation mechanisms and mechanisms for the balanced development of disciplines

After the emergence of COVID-19, global research on this disease was performed rapidly, and the research results were remarkable. However, the current research on COVID-19 does not show strong international scientific cooperation, and even some countries with a high incidence of COVID-19 lack external cooperation. Therefore, further strengthening of international cooperation in scientific research is still needed; countries should strengthen cooperation with the WHO and take the initiative to share information. In addition, despite the current interdisciplinary research cooperation model, the proportion of public health research achievements has decreased, indicating that the prevention and control of the global COVID-19 pandemic still require attention. The world should prioritize prevention and control and promptly establish a major epidemic prevention and control system. For example, the governments of all countries should establish a hierarchical prevention system, develop a strong monitoring and early warning system, and implement effective measures to prevent and control the spread of the epidemic. The strengthening of international cooperation in scientific research, an understanding of the global governance system, the formation of a pattern of interdisciplinary and balanced development, and the construction of a sound epidemic prevention and control system in a timely manner are crucial for the prevention and control of the epidemic.

\section{Strengthen the construction of scientific research platforms to eliminate the research gap as soon as possible} Although researchers have achieved some progress in the study of COVID-19, these studies are still in the preliminary exploratory stage, and gaps in some aspects of research still exist. For example, in terms of virus traceability, the natural host is known, but the route of transmission from the intermediate host to humans requires further study. Only by identifying the natural and intermediate hosts of the virus and understanding its transmission route as soon as possible will we be able to develop more targeted early warning, prevention and control strategies. In terms of clinical treatment, only the previous experience of using antivirals for pneumonia is currently used as a basis for treatment, and no specific drugs or methods for the diagnosis and treatment of COVID-19 have been identified. Therefore, improvements in effective diagnostic and treatment methods should be further investigated in future studies. We must strengthen the development of research platforms, improve the efficiency of research, and accelerate the development of therapeutic drugs and vaccines. 
Improve the mechanism of cooperation between scientific research and clinical treatment

As researchers develop a new understanding of COVID19 , the diagnosis and treatment guidelines issued by the WHO and other countries should be updated in a timely manner according to the scientific research results to provide faster and more effective diagnostic and treatment measures for front-line medical staff. Rapid and effective diagnostic and treatment methods will help reduce the burden on medical supplies and medical staff. At the same time, the WHO guidelines should be actively used. For example, countries should follow the WHO guidelines and provide corresponding prevention and treatment measures for relatively susceptible groups, such as pregnant women, children, the elderly and other special populations.

\section{Acknowledgements}

We would like to thank all authors, reviewers and editors for their critical discussion of this manuscript, and apologize to those individuals who were not mentioned due to limited space.

\section{Author contributions}

FY and SZ completed this manuscript and analyzed the data. JH, LW, XW and FP collected the data. SZ prepared Figs. 1, 2, 3, 4, 5, 6, QZ revised Figs. 1 and 5 FY, FX and QW conceived the study and participated in its design. All authors read and approved the final manuscript.

\section{Funding}

This work was supported by the China Postdoctoral Science Foundation (No. 2019M662373), the Spatiotemporal Epidemiology of COVID-19 (No. 2020SFXGFY02-2), the China Postdoctoral Science Foundation (No. 2019M662373), and the National Key Research and Development Program of China (No. 2020YFC2003500)

\section{Compliance with ethical standards}

\section{Conflict of interest}

We declare no competing interests.

\section{Author details}

${ }^{1}$ Department of Epidemiology and Biostatistics, School of Public Health, Cheeloo College of Medicine, Shandong University, Jinan 250012, Shandong,
China. ${ }^{2}$ Institute for Medical Dataology, Shandong University, Jinan 250002 , Shandong, China.

Received: 24 May 2020 Accepted: 12 September 2020

Published online: 30 September 2020

\section{References}

1. Bai Y, Yao L, Wei T, Tian F, Jin DY, Chen L, Wang M. Presumed asymptomatic carrier transmission of COVID-19. JAMA. 2020;323(14):1406-7.

2. Deutsch L, Houri I, Ben-Ari Z, Shlomai A, Veitsman E, Cohen-Ezra O, Issachar A, Mor O, Gozlan Y, Bruck R. Ombitasvir/paritaprevir/ritonavir \& dasabuvir \pm ribavirin following protease inhibitors failure-a prospective multi-centre trial. BMC Infect. Dis. 2020;20:264.

3. Du Z, Xu X, Wu Y, Wang L, Cowling B, Meyers L. The serial interval of COVID-19 from publicly reported confirmed cases. medRxiv. 2020. https ://doi.org/10.1101/2020.02.

4. Hui DS, Azhar IE, Madani T, Ntoumi F, Koch R, Dar O, et al. The continuing 2019-nCoV epidemic threat of novel coronaviruses to global health: the latest 2019 novel coronavirus outbreak in Wuhan, China. Int J Infect Dis. 2020;91:264-6.

5. Ji W, Wang W, Zhao X, Zai J, Li X. Cross-species transmission of the newly identified coronavirus 2019-nCoV. J Med Virol. 2020;92(4):433-40.

6. Li H, Wang Y, Xu J, Cao B. Potential antiviral therapeutics for 2019 novel coronavirus. Zhonghua jie he he hu xi za zhi = Zhonghua jiehe he huxi zazhi $=$ Chin J Tuberc Respir Dis. 2020;43:E002.

7. Salata C, Calistri A, Parolin C, Palu G. Coronaviruses: a paradigm of new emerging zoonotic diseases. Pathog Dis. 2019;77(9):ftaa006.

8. Song W, Gui M, Wang X, Xiang Y. Cryo-EM structure of the SARS coronavirus spike glycoprotein in complex with its host cell receptor ace2. PLoS Pathog. 2018;14(8):e1007236.

9. Wang H, Zhang L. Risk of COVID-19 for patients with cancer. Lancet Oncol. 2020;21(4):e181.

10. Wang M, Cao R, Zhang L, Yang X, Liu J, Xu M, Shi Z, Hu Z, Zhong W, Xiao $G$. Remdesivir and chloroquine effectively inhibit the recently emerged novel coronavirus (2019-nCoV) in vitro. Cell Res. 2020;30(3):269-71.

11. Wei J, Xu H, Xiong J, Shen Q, Fan B, Ye C, Dong W, Hu F. 2019 Novel coronavirus (COVID-19) pneumonia: serial computed tomography findings. Korean J Radiol. 2020;21(4):501-4.

Publisher's Note Springer Nature remains neutral with regard to jurisdictional claims in published maps and institutional affiliations. 